\title{
Comparison of electric dipole moments and the Large Hadron Collider for probing $C P$ violation in triple boson vertices
}

\author{
Sunghoon Jung ${ }^{1}$ and James D. Wells ${ }^{2,1}$ \\ ${ }^{1}$ Michigan Center for Theoretical Physics (MCTP), University of Michigan, Ann Arbor, Michigan 48109-1120, USA \\ ${ }^{2}$ CERN, Theory Division, CH-1211 Geneva 23, Switzerland \\ (Received 17 December 2008; published 15 July 2009)
}

\begin{abstract}
$C P$ violation from physics beyond the standard model may reside in triple boson vertices of the electroweak theory. We review the effective theory description and discuss how $C P$-violating contributions to these vertices might be discerned by electric dipole moments (EDM) or diboson production at the LHC. Despite triple boson $C P$-violating interactions entering EDMs only at the two-loop level, we find that EDM experiments are generally more powerful than the diboson processes. To give an example to these general considerations we perform the comparison between EDMs and collider observables within supersymmetric theories that have heavy sfermions, such that substantive EDMs at the one-loop level are disallowed. EDMs generally remain more powerful probes, and next-generation EDM experiments may surpass even the most optimistic assumptions for LHC sensitivities.
\end{abstract}

DOI: 10.1103/PhysRevD.80.015009

PACS numbers: 13.40.Em, 12.60.Cn, 13.40.Gp

\section{INTRODUCTION}

Cabibbo-Kobayashi-Maskawa (CKM) phases explain all observed $C P$ violations. However, baryogenesis apparently requires more $C P$-violation than is provided for by the standard model (SM). Thus, physics beyond the SM should contain new source of $C P$ violation that is somehow small enough not to be in conflict with experiment.

$C P$ violation from new physics can manifest itself in several ways. One way is by measuring an electric dipole moment (EDM) of a fermion. No EDM has been found to date. The current experimental electron EDM (eEDM) bound is $d_{e} \leq 2.14 \times 10^{-27} \mathrm{e} \mathrm{cm}$ at $95 \%$ C.L. [1], which already puts a strong constraint on physics beyond the SM. In supersymmetric theories [2], the eEDM induced at oneloop is usually larger than this bound so we need several assumptions [3-5] or cancellation mechanisms [6,7] to avoid this limit for a wide range of parameter space.

$C P$ violation can also be seen in $C P$ asymmetries of particle energy-momentum distributions at colliders. One such $C P$ asymmetric collider observable was proposed recently using the interference effect between $C P$-conserving and violating $W W Z$ interactions in the diboson production processes at LHC [8]. This observable may be able to improve collider sensitivities on $C P$-violating couplings such as triple boson vertices (TBV) by up to 2 orders of magnitude from the most recent LEP results. Since we expect that abundant diboson production will occur at LHC, and they have clean trilepton decay signals, this observable is useful to probe new physics at the LHC. This improvement raises the hope of discovery, and it is worthwhile studying the possible reach of both the collider observable and EDM measurements in more detail.

Intuition holds in the physics community that EDMs are the most powerful probes of new physics contributions to flavor-preserving $C P$ violation. That intuition is largely based on the varieties of supersymmetric theories that have dipole moments induced at the one-loop level. However, given the possibility of the LHC increasing the probing sensitivity by a few orders of magnitude, we investigate how solid that intuition is within the context of theories that have suppressed one-loop contributions to EDMs. Our primary example is supersymmetry with heavy sfermion masses. Ultimately, we shall not disagree that EDMs are unlikely to be supplanted by the LHC in the search for new sources of $C P$ violation. We detail the path to that strengthened conclusion below.

\section{TRIPLE BOSON VERTICES AND CP VIOLATING OBSERVABLES}

\section{A. Triple boson vertices effective interactions}

Diboson production channels at the LHC are described in Fig. 1 using the low-energy effective theory below the electroweak scale. This effective theory is obtained by integrating out heavy particles in physics beyond the SM. The modified SM interactions, which now contain both $C P$-even and odd interactions, are represented as small blobs in the figure. One can see from the figure that we should study the diboson production channels at the LHC, involving TBV $V V V, h V V$ and couplings with fermions $V f f, h f f$.

We will focus only on TBV among them. One reason for this is that we can easily extend our work to include fermion couplings without changing the conclusions. Secondly, $C P$-odd effective couplings are mediated by particles in the physics beyond the standard model and are loop suppressed. Any charged particle couples to the vector bosons, whereas only a small number of particles couple to a specific fermion typically. Therefore, TBV is more generally present than more direct $C P$-violating cou- 

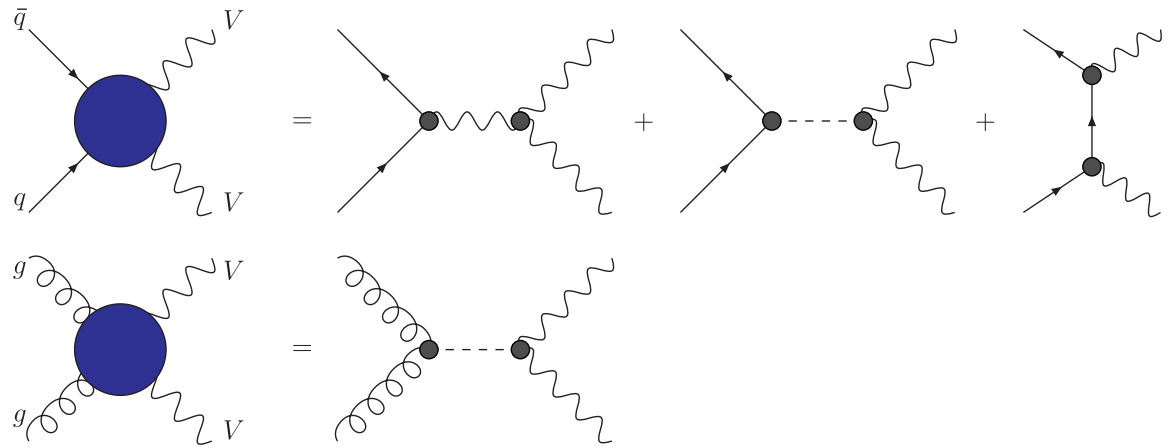

FIG. 1 (color online). Diboson production processes at the LHC. Blobs on the right-hand side are effective interactions in the lowenergy effective theory. These effective interactions contain both $C P$-even and odd contributions.

plings with fermions. In addition, large $C P$-odd $V f f$ couplings with $V=\gamma$ can induce an EDM without loop suppression, as is discussed in Sec. II C. Since other $V f f$ couplings are presumably related with the $\gamma f f$ coupling in an underlying theory, it is difficult to avoid the experimental EDM limit with large $C P$-odd $V f f$ couplings. Thus, a meaningful analysis can be carried out with TBV only.

The effective Lagrangian of $C P$-odd TBV is [9]

$$
\begin{aligned}
\mathcal{L}_{C P-\text { odd TBV }}= & i g_{W W V}\left(\tilde{\kappa}_{V} W_{\mu}^{+} W_{\nu} \tilde{V}^{\mu \nu}+\frac{\tilde{\lambda}_{V}}{m_{W}^{2}} W_{\nu}^{+\mu} W_{\rho}^{\nu} \tilde{V}_{\mu}^{\rho}\right. \\
& \left.+g_{4}^{V} W_{\mu}^{+} W_{\nu}\left(\partial^{\mu} V^{\nu}+\partial^{\nu} V^{\mu}\right)\right) \\
& +\frac{g M_{W}}{4}\left(g_{H_{i} W W} \tilde{\eta}_{i}^{W} \tilde{W}^{\mu \nu} W_{\mu \nu}^{+}\right. \\
& \left.+g_{H_{i} Z Z} \frac{\tilde{\eta}_{i}^{Z}}{2 c_{W}^{2}} \tilde{Z}^{\mu \nu} Z_{\mu \nu}\right) H_{i}
\end{aligned}
$$

where $g_{W W \gamma}=-e, g_{W W Z}=-e \cot \theta_{W}$, and $g_{H_{i} V V}$ is the ratio of $C P$-even $H_{i} V V$ coupling to SM $H_{i} V V$ coupling. $V$ can be $\gamma$ or $Z$. $V^{\mu \nu}=\partial^{\mu} V^{\nu}-\partial^{\nu} V^{\mu}$ and likewise for $W^{\mu \nu}$. Index $i$ runs for two light ( $C P$-even) Higgses. $g_{4}^{V}$ is $\mathrm{C}$ odd, while others are $\mathrm{P}$ odd, so $g_{4}^{V}$ is not relevant for our work as discussed in Sec. II B. Higher dimensional operators are suppressed by the electroweak scale $M_{W}$. Higgs couplings to photons and gluons can also be written in the same way. These effective couplings are actually momentum dependent. However, we can reasonably choose to study constant on-shell couplings as argued in Appendix A.

It is useful to know the $S U(2) \times U(1)$ invariant dimension-six operators that generate the effective triple gauge couplings in Eq. (1) after electroweak symmetry breaking. $H^{\dagger} H V^{\mu \nu} \tilde{V}_{\mu \nu}$ and $D_{\mu} H^{\dagger} T^{a} D_{\nu} H \tilde{V}^{a \mu \nu}$ generate $\tilde{\kappa}_{V} . \tilde{\lambda}_{V}$ is generated by $\epsilon_{a b c} \tilde{W}_{\nu}^{a \mu} W_{\rho}^{b \nu} W_{\mu}^{c \rho}$, which does not involve Higgs fields. $V_{\mu \nu}$ and $W_{\mu \nu}$ here are full field strengths. $C P$-odd neutral $V V V$ couplings are not generated by these operators. As couplings with photons and couplings with $Z$ bosons are presumably related in an underlying theory, we shall reduce redundancy and give results in terms of the $Z$ boson coupling only.

\section{B. $C P$ asymmetric collider observable}

$C P$ asymmetries at colliders are observables well known to probe $C P$-violating interactions [10]. It has been shown that if absorptive SM backgrounds are known well, the LHC may be sensitive to $\tilde{\lambda}_{Z}$ coupling perhaps as low as the $\tilde{\lambda}_{Z} \lesssim 0.001$ with $100 \mathrm{fb}^{-1}$, which would be a significant improvement over LEP2 capabilities, for example [8]. This sensitivity was achieved based on the fact that the cross section proportional to the $\epsilon_{\mu \nu \rho \sigma}$ tensor is a signal of the $C P$ violation since the tensor is odd under time reversal. Thus, only $P$ and $C P$-odd couplings in Eq. (1) are potentially able to be probed with this precision. Although no equivalent small value has been estimated for $\tilde{\kappa}_{Z}$, we shall suggest by analogy to $\tilde{\lambda}_{Z}$ that it may be possible. The $C$-odd coupling $g_{4}^{V}$ can be probed in other ways and will not be treated in this paper.

$\mathrm{CP}$-violating Higgs couplings can also be probed at the LHC in the same way, in principle. Several other collider observables sensitive to Higgs couplings have been studied as well based on the angular distributions of final leptons. The sensitivities on the $C P$-violating $h Z Z$ coupling are usually expected to be around $\tilde{\eta}^{Z} \lesssim O(0.1)$ with $100-300 \mathrm{fb}^{-1}$ of data from the process $h \rightarrow Z Z \rightarrow 4 l$ at LHC, and possibly $O(0.01)$ from Higgsstrahlung at a future $e^{+} e^{-}$linear collider [11]. As we study EDM sensitivities to the $C P$-violating couplings involving the Higgs boson, we compare results to the $\tilde{\eta}^{Z} \lesssim O(0.1)$ LHC expected sensitivity.

\section{Electric dipole moments}

One and two-loop order generation of EDMs are shown in Fig. 2 using the effective theory. $C P$-odd effective couplings in the effective theory are represented as small blobs in the figure. Since effective $\mathrm{CP}$-odd couplings are generated at loop order, the tree-level diagram on the righthand side implies a one-loop contribution to the EDM, and others are two-loop contributions.

We want to avoid one-loop-induced EDM in our study. The first reason for this is that a one-loop-induced EDM is usually larger than experimental bounds in many models. 


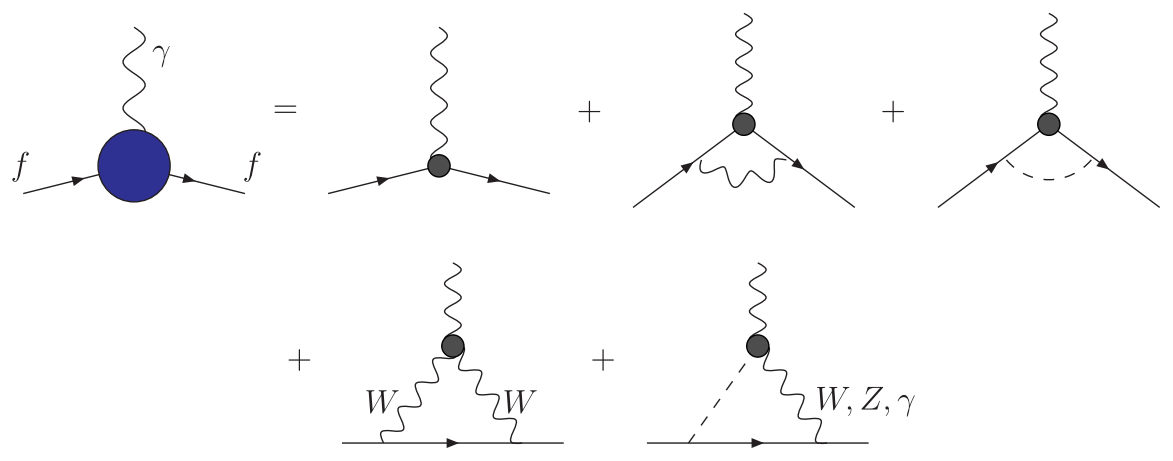

FIG. 2 (color online). EDM diagrams at one- and two-loop orders with the effective $C P$-odd couplings represented as small blobs. Since effective couplings are radiatively generated, the first diagram on the right-hand side is a one-loop contribution and others are two-loop.

Secondly, we want to give a "one-loop advantage" to collider observables-we expect that TBVs are generated at one-loop order, which only then enables EDMs at twoloop order. As can be seen from the figure, one-loop EDM (first diagram) corresponds to $C P$-odd $V f f$ couplings with $V=\gamma$ and on-shell external particles. The suppression of the one-loop induced EDM roughly implies the smallness of $C P$-odd $V f f$ couplings, and vice versa. Then the twoloop diagrams in the first line of Fig. 2 are also suppressed.

We will consider only two-loop contributions in the second line of Fig. 2 with specified insertions of effective couplings. We note that these effective interactions are $C P$-violating TBVs that were necessary for the diboson production process as discussed in Sec. II A. At two-loop order, $W W$ and scalar-vector can transmit $C P$ violation to SM fermions, whereas scalar-scalar mediation is very small due to small Yukawa couplings. Only $W W$ can mediate $C P$ violation without Higgs bosons because there is no $C P$-odd triple neutral electroweak boson couplings. EDMs are generated only through these $C P$-violating TBVs as long as we ignore quartic and higher effective couplings. It can also be inferred that EDMs and $C P$-violating TBVs depend on the same $C P$ phases as will be discussed in Sec. III A.

The electric dipole operator should be renormalization group evolved from high scale down to the fermion mass scale at which the fermion EDM is defined. Renormalization group flow mixes this operator with other operators with the same quantum numbers such as chromoelectric dipole, three-gluon Weinberg operator [12], and $S U(2)$ analogies of these. For eEDM, not all are relevant since the electron is colorless. The remaining $S U(2)$ operators are relatively suppressed by multiple powers of $g / g_{S}$, and the QED renormalization effects are smaller than QCD. We will not consider renormalization effects for eEDM.

In this paper we focus on the electron EDM since the experimental measurements are excellent and improving, and the theory computation has minimal theoretical uncertainty. Of course, one expects a high degree of correlation of one EDM to other EDMs in most theories of physics beyond the SM, and later we shall briefly study the correlation of electron EDM and neutron EDM. As stated earlier, the current sensitivity limit on the eEDM is $d_{e} \leq 2.14 \times 10^{-27} \mathrm{e} \mathrm{cm}$ at $95 \%$ C.L. [1]. Upon surveying the literature, one expects that the future eEDM sensitivity of the near-term future experiments to be approximately $10^{-29}$ e cm [13-15]. When appropriate, we shall use these numbers as benchmark sensitivities in the numerical discussion ahead.

\section{COMPARISON IN SUPERSYMMETRIC MODELS}

Now we work on specific supersymmetric models. In trying to find scenarios where the LHC can probe better the new $C P$-violating physics compared to eEDM measurements, we will work on models in which the eEDM is twoloop suppressed, while TBV is only one-loop suppressed. As the simplest possibility we study the split supersymmetry limit where all scalars except SM-like neutral Higgs are heavy and decoupled [16-19]. Another possibility is to take only first two generations of sleptons and squarks to be heavy, allow $C P$-violating couplings in the trilinear scalar vertices of the third generation, which induces radiative breaking of $C P$ invariance in the Higgs sector. The mixing of $C P$-even and $C P$-odd eigenstates in the Higgs sector gives opportunity to colliders to discover these new sources of $C P$ violation.

\section{A. Physical $C P$ phases and triple Boson vertices}

One can see the relevance of TBVs in supersymmetric models in a more useful way using the physical $C P$ phases. Using $R$ and Peccei-Quinn (PQ) symmetries, it is shown that in any phase conventions there are two sets of physical $C P$ phases in the universality ansatz [20,21]: $\arg \left(A \mu b^{*}\right)$, $\arg \left(M_{1,2,3} \mu b^{*}\right)$, where parameters are the usual soft supersymmetry parameters and Higgsino mass $\mu$. The $\arg \left(M_{i} M_{j}^{*}\right)$ are also allowed by the same argument. Since we impose grand unified theory-like relations on gaugino 
masses these phases are not relevant to consider. As lowenergy effective operators composed of SM Dirac fermions and vector fields are neutral under $R$ and PQ symmetries, we can argue that low-energy physical observables should depend on the above combinations, which are the only $R$ and PQ invariants. Indeed, both $R$ and PQ are needed and enough for us to do that because all complex soft phases are charged under at least one of them. This argument does not restrict soft squark/slepton masses.

Since the $b$ term appears only in the Higgs sector, $C P$ violation in the soft supersymmetry breaking sector can be transferred to low-energy effective operators consisting of Higgs bosons at one-loop order. Possible $C P$-violating interactions with one SM Higgs field are Higgs-vectorvector, Higgs-fermion-fermion, and Higgs-scalar-scalar couplings. Higgs-Higgs-vector coupling is usually related to the Higgs-vector-vector via the underlying theory. As sizable tree-level processes at LHC involve at most two Higgs bosons, the scalar quartic coupling is not relevant.

As discussed in Sec. II A, there are also $S U(2) \times U(1)$ invariant dimension-six operators composed of Higgs bosons and vectors. After the Higgs bosons get vacuum expectation values, these operators can induce effective triple gauge couplings $W W V$, where $V$ is a neutral vector boson. Thus, $C P$-violating TBVs are not only relevant but also can indeed be generated at one-loop order in supersymmetric models. It is also clear that $C P$-violating TBVs and EDMs depend on the same $C P$ phases.

\section{B. Supersymmetry with heavy sfermions}

The split sfermion/ino limit of supersymmetry (split supersymmetry) does not naturally induce large EDMs. In this limit, charginos and neutralinos are not decoupled, and they carry $C P$ phases in the soft supersymmetry breaking sectors. These ino sectors couple to SM fermions at tree level only via ino-fermion-sfermion couplings, which lead to suppressed amplitudes in split supersymmetry due to the heavy sfermions. So $C P$ violation in the SM fermion sector, e.g., EDM, are induced beginning at two-loop order. Recent studies have shown that the electron EDM turns out to be generically smaller than or around the current limit in most of parameter space even with maximum $C P$ phases $[22,23]$.

To compute the effects, the input parameters are $\mu, \tilde{M}_{1,2}$ and their phases, $\tan \beta$ and SM-like neutral Higgs mass $M_{h}$. The sign of $\mu$ is not relevant as it just shifts the $C P$ phase by $\pi$. Since we are interested in electron electric dipole moments, the gluino mass $M_{3}$ is not relevant. Once we assume grand unified theory-like relation between gaugino masses, only one $C P$ phase $\arg \left(\tilde{M} \mu b^{*}\right)$ is physical. The phase of $b$ is related to the relative phase of $H_{u}$ and $H_{d}$ via the minimization condition of the Higgs potential; $b /\left(v_{u} v_{d}\right)$ is real at tree level. We will work in the basis in which $b$ is real, then the two Higgs bosons have opposite phases. $U(1)_{Y}$ rotations of $H_{u}$ and $H_{d}$ can remove this relative phase, and the Higgs boson vacuum expectation value (vevs) are real in the same basis [2]. The only physical combination of $C P$ phases remaining is $\arg (\tilde{M} \mu)$. It is clear that these $C P$ phases reside in the chargino and neutralino sectors.

EDMs in split supersymmetry have been computed in previous works [22-24]. We also compute the effective $C P$-odd TBVs generated by diagrams shown in Fig. 3(a), and apply them to the eEDM and collider observables. We give supporting analytic results in the Appendix B.

One interesting result to notice is that the $\tilde{\lambda}_{V}$ coupling is not generated at one-loop. It is simply because this coupling is generated by a dimension-six operator that does not involve Higgs fields as discussed in Sec. II A, whereas the physical $C P$ phase depends on the $b$ term. We can see this more explicitly in terms of current eigenstates depicted in Fig. 3(b). As Higgs-Higgsino-gaugino coupling couples gaugino and Higgsino, either a gaugino or a Higgsino runs in the loop without Higgs. Then $\mu$, as an interaction between $\tilde{H}_{u}$ and $\tilde{H}_{d}$, and $\tilde{M}_{1,2}$ cannot appear together, and hence no $C P$ phase. (Recall that the physical phases are $\mu M_{1,2}$ in our basis.) Indeed, the diagram with only gaugino (or Higgsino) is proportional to $|\tilde{M}|^{2}$ (or $|\mu|^{2}$ ) because of the charge flow direction as shown in the figure. These are real, i.e., no $C P$ violation.

Both $C P$-violating TBVs and eEDM are approximately proportional to $\sin 2 \beta$ by essentially the same reason. To see this it is again easiest to think in terms of current eigenstates. Relevant diagrams are then Fig. 3(b) with the $W$ boson on top replaced by a neutral gauge boson, and with mass insertions replaced by external $H_{u}, H_{d}$ legs and their vevs. Note that we need one $H_{u}$ and one $H_{d}$ in order to insert both $\tilde{M}$ and $\mu$. As we take neutral Higgs fields other than SM-like Higgs boson to be very heavy, we obtain a simple relation between Higgs mixing angle $\alpha$ and vev ratio $\beta: \tan \alpha=\tan \beta$ at leading order. Therefore, each vev of $H_{u}$ and $H_{d}$ carries $\sin \beta$ and $\cos \beta$, respectively, hence $\sin 2 \beta$ overall. eEDM is generated by inserting these effective interactions in Fig. 2, thus having the same $\sin 2 \beta$ dependence.

We now look at some numerical results for this scenario. eEDM and $C P$-violating TBVs depend on input parameters quite similarly. $\tan \beta$ dependence cancels when we study the relative importance of eEDM and collider observables as we saw above. Heavy $M_{h}$ can suppress the eEDM since Higgs boson mediated two-loop eEDM dominates numerically in this scenario, while TBVs are independent of $M_{h}$. However, due to the narrow consistent Higgs mass range $115 \mathrm{GeV} \leq M_{h} \lesssim 150 \mathrm{GeV}$ of the light SM-like Higgs boson in supersymmetry, this suppression is not very significant. $M_{1}$ dependence is weak since the bino does not couple to gauge bosons at tree-level. Dependence on the remaining gaugino/Higgsino mass parameters can be different because the eEDM is two-loop, while TBVs are one-loop physics. 
We choose to draw plots in $M_{2}-\mu$ plane. In Fig. 4, we show eEDM and $C P$-violating TBVs in this plane. We set $\tan \beta=1$, which is not allowed, because this small $\tan \beta$ induces too large Yukawa coupling, but one can extrapolate the results linearly with $\sin 2 \beta$ as discussed above. In almost all of the parameter space, the current eEDM limit and the expected collider observable are not sensitive enough to probe $C P$ violations in split supersymmetry even with maximum $C P$ phases.

Then the next question is if there exists parameter space in which eEDM is well below the future sensitivity while TBVs are around the future reach. The answer is (almost) no. In order to see this we scatter input parameters randomly within the following range:

$$
\begin{aligned}
100 \mathrm{GeV} & \leq M_{1,2}, \mu \leq 1000 \mathrm{GeV}, \\
115 \mathrm{GeV} & \leq M_{h} \leq 180 \mathrm{GeV}, \quad 2 \leq \tan \beta \leq 50 .
\end{aligned}
$$

If $M_{2}, \mu$ are a few $\mathrm{TeV}$, then both eEDM and collider observable are well below the current sensitivities as can be seen in Fig. 4, so we now focus on the sub-TeV gaugino/ Higgsinos. In addition, as stated earlier, we identify the future eEDM sensitivity to be $10^{-29}$ e cm for Refs. [13$15]$.

In Fig. 5, we see that the eEDM and TBVs are closely related so that there is a narrow allowed region of eEDM for each specific TBV value, and vice versa. The Higgs boson coupling shows stronger correlation with the eEDM due to dominance of the Higgs-mediated eEDM over $W W$-mediated eEDM. This correlation is what we expected based on the observation that any $C P$-violating TBV can induce an eEDM discussed in Sec. II C.

For $d_{e}<10^{-29} \mathrm{e} \mathrm{cm}$, which is just below the reference point of future eEDM measurement sensitivity, $C P$-violating TBV values correspond to $\tilde{\eta}^{Z}, \tilde{\kappa}^{Z} \lesssim$ $8 \times 10^{-6}$. Although it remains to be seen how well dedicated LHC experiments can do, if other $C P$-violating observable expectations are a rough guide it is unlikely that these couplings can be probed at the one part per mil level at the LHC. If LHC fails to reach that very high sensitivity, the proposed eEDM sensitivity of $\sim 10^{-29} \mathrm{e} \mathrm{cm}$ would be a more powerful probe of $C P$ violation from new physics.

The neutron EDM is also precisely measured with the current sensitivity [25] $d_{n}<6.3 \times 10^{-26} \mathrm{e} \mathrm{cm}$, and can be improved in the future. This can be a competitor to the eEDM measurement depending on the future improvement and the theory prediction of the neutron EDM. In split supersymmetry, we compare computed eEDM and neutron EDM in Fig. 6. The neutron EDM is generated by constituent quark EDMs induced by the same types of diagrams generating eEDM because heavy squarks suppress chromo-electric dipole and three-gluon Weinberg operators. Thus, the neutron EDM depends on the same $C P$ phases as eEDM does, and is closely related to eEDM as can be seen in Fig. 6 [26].

\section{MSSM with radiative breaking of Higgs sector $C P$ invariance}

We relax the split limit but keep the first two generations of squarks and sleptons to be very heavy to avoid large flavor changing neutral current and one-loop induced EDM $[3,27]$. There are now not only additional physical $C P$ phases, but the $C P$ invariance of the Higgs sector can be radiatively broken so that there might be less correlation between eEDM and $C P$-violating TBVs.

In the low-energy effective theory, we have two more neutral Higgs bosons, charged Higgs bosons and a third generation of squarks and sleptons in addition to split limit field contents. As a trilinear $A$-term interaction with stop (in large extent with sbottom) becomes relevant, the physical $C P$ phases $\arg \left(A \mu b^{*}\right)$ cannot be ignored. These $C P$ phases induce $C P$ violation in the two-point Green's function through squark and quark loops, and mix $C P$-even and odd Higgs eigenstates [28,29]. Because of these loopinduced interactions, we call this "radiative breaking" of $C P$ invariance in the Higgs sector. One consequence of this important to us is that the pseudoscalar Higgs interactions
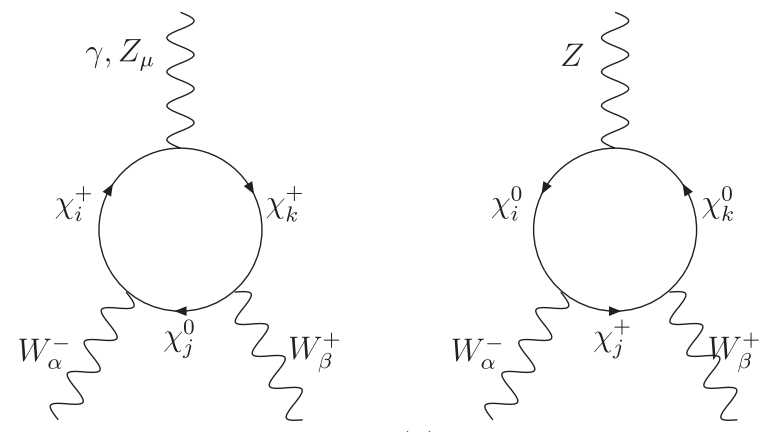

(a)

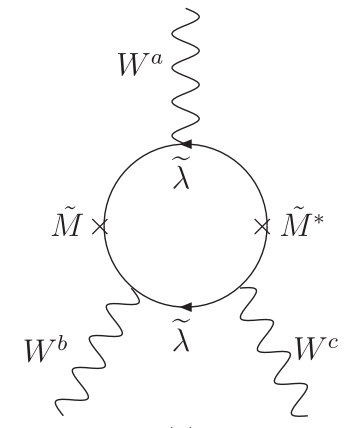

(b)

FIG. 3. (a) $C P$-odd TBV diagrams mediated by charginos $\chi_{i}^{+}(i=1,2)$ and neutralinos $\chi_{i}^{0}(i=1$, 4$)$. Similar diagrams generating $h V V$ couplings can also be drawn. (b) Diagram that is responsible for the $\tilde{\lambda}_{V}$ coupling is shown in terms of current eigenstates. Gaugino $\tilde{\lambda}$ is running in the loop, and its complex soft mass insertion is denoted as a cross. A similar diagram in which Higgsinos are running with mass $\mu$ insertions can also be drawn. 

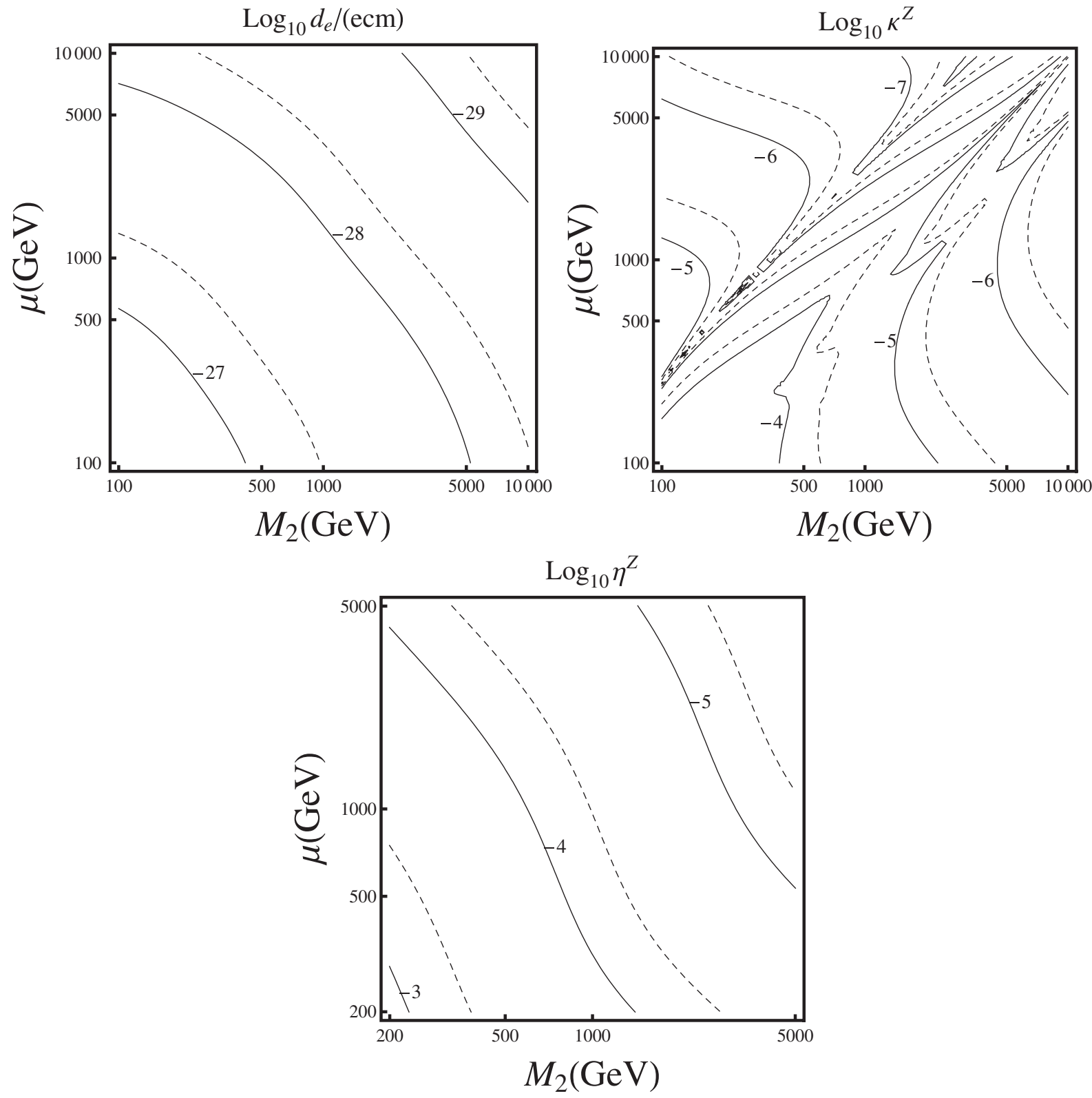

FIG. 4. Contour plots of eEDM, triple vector coupling $\tilde{\kappa}^{Z}$ and the Higgs coupling $\tilde{\eta}^{Z}$ to the $Z$ boson in the $M_{2}-\mu$ plane. $\log _{10}$ values are written on the solid contour lines. To facilitate rescaling by the reader, contours are made for $\tan \beta=1$ with maximum $C P$ phases. $M_{h}=120 \mathrm{GeV}$ is used. Abrupt changes of $\tilde{\kappa}^{Z}$ in the diagonal region are partially due to a change of sign.

with fermions generate $\mathrm{CP}$-violating $\mathrm{TBV}$ at one-loop order. Of course, TBVs induce EDMs, and the tension between them still exists.

However, the radiative breaking of $C P$ invariance can enhance the $C P$-violating collider observables. The neutral Higgs mass mixing matrix $O$ is defined as

$$
\left(\begin{array}{c}
H_{d} \\
H_{u} \\
A
\end{array}\right)=O\left(\begin{array}{l}
H_{1} \\
H_{2} \\
H_{3}
\end{array}\right),
$$

with $M_{H_{1}}<M_{H_{2}}<M_{H_{3}} . H_{1}\left(H_{2}\right)$ becomes the light (heavy) $C P$-even Higgs in the absence of $C P$ violation.
The scalar-pseudoscalar transitions $O_{A i}$ induce pseudoscalar couplings between Higgs boson $H_{i}$ and quarks. Therefore, $\mathrm{CP}$-violating $\mathrm{H}_{i} V V$ couplings generated by quarks are proportional to $O_{A i}$ as explicitly shown in Eq. (A18). Other mixing elements modify $C P$-conserving $H_{i} V V$ couplings. The ratio of the $C P$-even $H_{i} V V$ coupling in this scenario to the $\mathrm{SM} H_{i} V V$ coupling is written as [30]

$$
g_{H_{i} V V} \equiv c_{\beta} O_{H_{d} i}+s_{\beta} O_{H_{u} i} \text {. }
$$

As scalar-pseudoscalar mixing $O_{A i}$ increases, $g_{H_{i} V V}$ decreases because the mixing matrix is normalized. Thus, the ratio of the $C P$-odd Higgs couplings to $C P$-even Higgs 

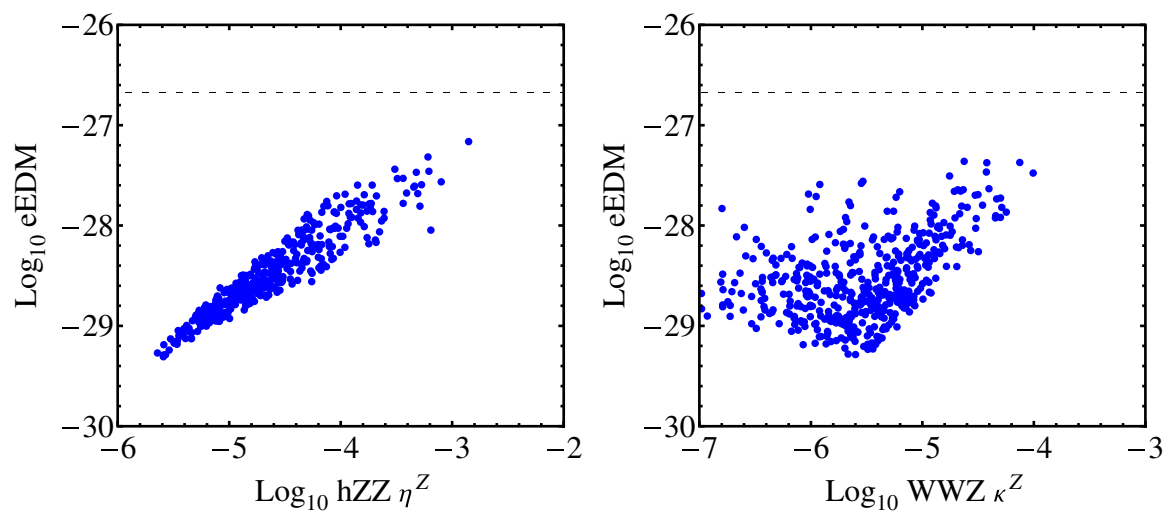

FIG. 5 (color online). $\quad C P$-violating Higgs (left) coupling $\tilde{\eta}^{Z}$ and triple vector (right) couplings $\tilde{\kappa}^{Z}$ to the $Z$ boson are plotted against eEDM in split supersymmetry. Input parameters are randomly scattered within the range Eq. (2). The dashed horizontal line represents the current experimental eEDM bound $d_{e}<2.14 \times 10^{-27} \mathrm{e} \mathrm{cm}$.

couplings can be relatively enhanced; i.e., the collider observable can be larger than what is expected in the case of no $C P$ even-odd mixing. It is interesting to study if this enhancement can win over the limited amount of $C P$ violation allowed due to the eEDM bound.

$C P$ even-odd mixing is large between two heavy neutral Higgs states $\mathrm{H}_{2}$ and $\mathrm{H}_{3}$, while the lightest Higgs $\mathrm{H}_{1}$ remains mostly $C P$ even [31]. So the enhancement is larger for $H_{2,3}$ couplings than for $H_{1}$. Meanwhile, as $g_{H_{i} V V}$ decreases we have to worry about a decrease of the cross section of diboson production mediated by Higgs bosons in Fig. 1. We focus on the $g g \rightarrow H_{i} \rightarrow Z Z \rightarrow 4 l$ diboson production channel for Higgs couplings collider observable as mentioned in Sec. II B. In order to use a collider observable, we need to be able to obtain at least a certain number of asymmetric events at the LHC. From this point of view, $H_{1}$ is a more important contributor than heavy Higgs bosons because $H_{1}$ is lighter and has larger couplings to the SM states. For example, the heavy Higgs $\mathrm{H}_{3}$, which becomes a $C P$-odd eigenstate in the limit of no $C P$ violation, usually has very small $\mathrm{CP}$-even $\mathrm{H}_{3} V V$ couplings, so there is little hope to measure them.

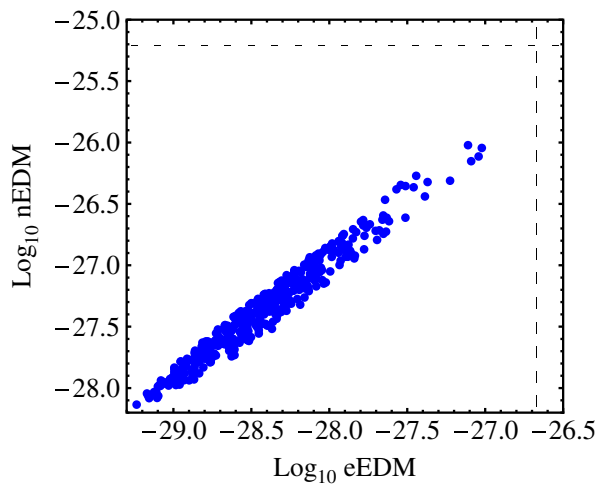

FIG. 6 (color online). Scatter plot of electron EDM and neutron EDM in split supersymmetry. Input parameters are scattered within the range Eq. (2). Dashed lines represent the current experimental sensitivities.
We now discuss the computation and numerical results for this scenario. $C P$-violating couplings are generated by Barr-Zee type diagrams in analogy to Fig. 3(a). In addition to gauginos and Higgsinos, the third generation squarks and quarks can run in the loop [32]. However, complex squark mixing angles cancel between adjacent vertices so squarks contribute to TBVs only at higher order. Top and bottom quarks can now generate $C P$-violating Higgs couplings through tree-level pseudoscalar coupling. Meanwhile, the triple vector couplings are not affected by quarks, and not very different from the split supersymmetry case. Thus, we focus on Higgs couplings in this section. Analytic results of quark and -ino contributions are shown in Appendix B. The complete set of two-loop induced EDMs in supersymmetry are computed in [32-34] and references therein.

$\tilde{\lambda}_{V}$ couplings are still not generated at one-loop order in our analysis. Physical $C P$ phases $\arg \left(A \mu b^{*}\right)$ and $\arg \left(M \mu b^{*}\right)$ depend on the $b$ term, so the same argument in split supersymmetry case that forbade $\tilde{\lambda}_{V}$ applies here as well. We can take another linear combination $\arg \left(A M^{*}\right)$, which appears at two-loop order, as squark and gaugino couple through a triple vertex with a quark. This $S U(2)$ analogy of the three-gluon Weinberg operator has little effect on the eEDM, as discussed in Sec. II C.

We assume the universality and flavor diagonality of soft masses and the trilinear coupling $A$ term for simplicity. The input parameters are then

$$
\begin{gathered}
M_{1,2}, \quad \mu, \quad \tan \beta, \quad M_{H^{ \pm}}, \\
A=A_{t}=A_{b}=A_{\tau}, \\
M_{\mathrm{SUSY}}=M_{Q_{3}}=M_{t}=M_{b}=M_{L_{3}}=M_{\tau}
\end{gathered}
$$

and soft $C P$ phases. As heavy Higgs bosons are not decoupled, the Higgs boson mixing angle $\alpha$ is not trivially related to vev ratio $\beta$, i.e., $\tan \alpha \neq \tan \beta$. The Higgs boson mixing angle now depends on various input parameters. Then the previous argument about $\sin 2 \beta$ dependence in 
split supersymmetry does not apply here. Indeed, several authors have shown that the eEDM increases overall with $\tan \beta$ [32]. Here, $\tan \beta$ rather plays the role of determining the amount of enhancement through $g_{H_{i} V V}$ and couplings with fermions, which can also be seen in Eq. (A18).

We have modified the $C P$ SUPERH 2.0 program [35] for numerical study. We scattered input parameters within the range

$$
\begin{aligned}
300 \mathrm{GeV} & \leq A, M_{\text {SUSY }} \leq 2000 \mathrm{GeV}, \\
130 \mathrm{GeV} & \leq M_{H^{ \pm}} \leq 250 \mathrm{GeV}, \\
150 \mathrm{GeV} \leq M_{1,2} \mu & \leq 1000 \mathrm{GeV}, \quad 2 \leq t_{\beta} \leq 50 .
\end{aligned}
$$

We also consider the following consistency condition:

$$
M_{H_{1}} \geq 115 \mathrm{GeV} \text {. }
$$

The light Higgs boson $H_{1}$ coupling to the $Z$ boson [see Eq. (1) for definition] versus the computed eEDM is shown in Fig. 7. Sample points that satisfy the consistency condition in Eq. (7) are represented as red circles. The eEDM measurement alone eliminates most of the sample points and restricts the Higgs coupling to be well below the experimental sensitivity $\sim O(0.1)$. Actually, in most of parameter space consistent with condition Eq. (7) and eEDM bound, $g_{H_{1} V V} \sim 1$ and $O_{A 1} \lesssim O(0.01)$. Thus, enhancement is too small to overcome the eEDM constraint. Large $C P$ violation needed to obtain large $O_{A 1}$ and small $g_{H_{1} V V}$ is still prohibited by the eEDM constraint.

For heavy Higgs boson $\mathrm{H}_{2}$, it also turned out to be very pessimistic for collider signatures of $C P$ violation. The required cross section just to discover the $\mathrm{H}_{2}$ Higgs boson itself almost eliminates the possibility for us to measure a

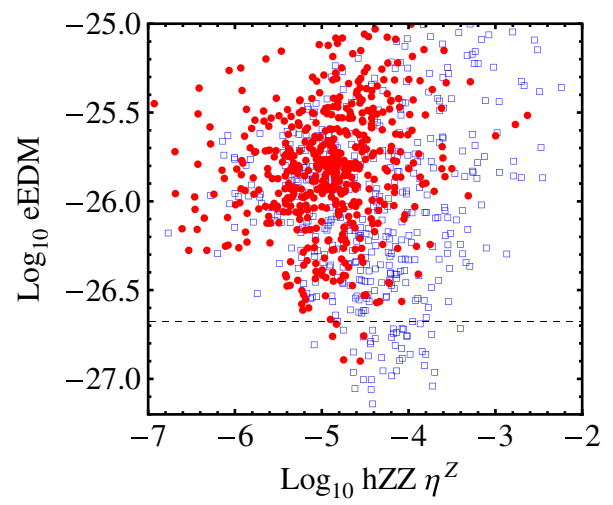

FIG. 7 (color online). Light Higgs $H_{1} C P$-violating coupling $\tilde{\eta}^{Z}$ to the $Z$ boson is plotted against eEDM with light third generation squarks. Blue $\square$ are excluded by $M_{h} \geq 115 \mathrm{GeV}$ while red satisfy this condition. Dashed line represents current eEDM bound $d_{e}<2.14 \times 10^{-27}$ e cm. Expected sensitivity on Higgs coupling is too large to be shown. This plot is generated with maximum $C P$-violating phases, and all points move downward as the phase angles decrease.

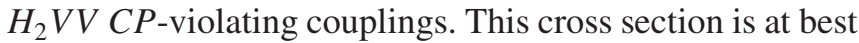
a few hundred $a b$ for $M_{H_{2}} \sim 170 \mathrm{GeV}$.

In this particular limit, the neutron EDM is usually predicted to be about 2 orders of magnitude larger than the eEDM, and hence is a stronger constraint on new physics [26]. This is mainly because the large $A_{t}$ coupling generates a three-gluon Weinberg operator that dominantly contributes to the neutron EDM, while the stop contribution to eEDM is subdominant. In any case, large $C P$ violations generating $C P$-violating $\mathrm{TBV}$ s eventually induce EDM, which is generally more constraining than $C P$-violating collider physics observables.

In concluding this section, we mention the previous work of Babu et al. [36], which had similar goals of comparison as this work. We briefly discuss that paper since it strengthens our conclusion. They found that oneloop lepton EDM mediated by slepton and gaugino/ Higgsino puts a severe constraint on one-loop generated $C P$-violating Higgs-lepton-lepton couplings in the minimal supersymmetric standard model (MSSM). So they sought other places where $C P$-violating Higgs boson couplings may be enhanced while the lepton EDM is relatively not. They noted that there is a tree-level $C P$ even-odd mixing in the Higgs sector of the next-to-minimal supersymmetric standard model. Since this $C P$-violating coupling is not loop suppressed and has different dependence on input parameters than the one-loop lepton EDM, they suggested that this would be a good place to observe large $C P$-violating Higgs couplings. However, once Higgsmediated two-loop EDM contributions are considered this conclusion must be modified. The large $C P$-violating Higgs boson couplings induce a two-loop EDM regardless of the origin of such $\mathrm{CP}$-violating couplings, which constrains the size of these Higgs boson couplings quite severely.

\section{CONCLUSIONS}

Our basic conclusion, which is supported by detailed investigations of various candidate theories that had a chance to contravene it, is this: Whatever the origin may be of $C P$-violating triple boson vertices, they induce EDMs, and although the physics that induces EDMs is "one loop down" compared to collider $C P$ asymmetries, the EDM experiments are sufficiently precise that they overcome the loop factor and are generally more powerful probes. We expect this conclusion to strengthen into the foreseeable future as EDM experiments become more sensitive.

\section{ACKNOWLEDGMENTS}

We thank R. Akhoury, J. Kumar, A. Leanhardt, A. Pierce, A. Rajaraman, and J. Shao for helpful discussions. This work is supported in part by the Department of Energy. S. Jung is also supported in part by Samsung. 


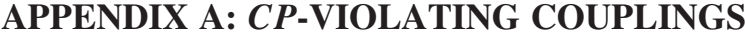

In this appendix, we present our conventions and analytic results of $C P$-violating TBVs.

\section{Conventions}

Gaugino and Higgsino masses are given as

$$
-\mathcal{L}=\frac{1}{2} M_{1} \tilde{B} \tilde{B}+\frac{1}{2} M_{2} \tilde{W}^{a} \tilde{W}^{a}+\mu \tilde{H}_{u} \epsilon \tilde{H}_{d} .
$$

Chargino, neutralino mixing matrices $U, V, N$ satisfy

$$
N^{*} M_{\chi^{0}} N^{\dagger}=M_{D^{0}}, \quad U^{*} M_{\chi^{+}} V^{\dagger}=M_{D^{+}},
$$

where $M_{\chi^{0}}$ and $M_{\chi^{+}}$are as in Ref. [2]. The subscript D implies a diagonal matrix with positive elements.

The interaction Lagrangian of split supersymmetry in terms of mass eigenstates is

$$
\begin{aligned}
\mathcal{L}= & g \bar{\chi}_{i}^{0} \gamma^{\mu}\left(C_{i j}^{L} P_{L}+C_{i j}^{R} P_{R}\right) \chi_{j}^{+} W_{\mu}^{+}+\text {H.c. }+\frac{g}{c_{W}} \bar{\chi}_{i}^{+} \gamma^{\mu}\left(F_{i j}^{L} P_{L}+F_{i j}^{R} P_{R}\right) \chi_{j}^{+} Z_{\mu}+\frac{g}{c_{W}} \bar{\chi}_{i}^{0} \gamma^{\mu}\left(H_{i j}^{L} P_{L}+H_{i j}^{R} P_{R}\right) \chi_{j}^{0} Z_{\mu} \\
& +\frac{g}{\sqrt{2}} \bar{\chi}_{i}^{+}\left(D_{i j}^{L} P_{L}+D_{i j}^{R} P_{R}\right) \chi_{j}^{+} h+\frac{g}{\sqrt{2}} \bar{\chi}_{i}^{0}\left(D_{i j}^{\prime L} P_{L}+D_{i j}^{\prime R} P_{R}\right) \chi_{j}^{0} h-e Q_{f} \bar{f} \gamma^{\mu} f A_{\mu}+\frac{g m_{f}}{2 M_{W}} \bar{f} f h,
\end{aligned}
$$

where $f$ is a fermion for which the EDM is calculated. C,D,F, and $\mathrm{H}$ are give by

$$
\begin{gathered}
C_{i j}^{L}=N_{i 2} V_{j 1}^{*}-\frac{1}{\sqrt{2}} N_{i 4} V_{j 2}^{*}, \quad C_{i j}^{R}=N_{i 2}^{*} U_{j 1}+\frac{1}{\sqrt{2}} N_{i 3}^{*} U_{j 2} \quad F_{i j}^{L}=-\delta_{i j} c_{W}^{2}+\frac{1}{2} V_{i 2} V_{j 2}^{*}, \\
F_{i j}^{R}=-\delta_{i j} c_{W}^{2}+\frac{1}{2} U_{i 2}^{*} U_{j 2} \quad H_{i j}^{L}=-\frac{1}{4}\left(N_{i 3}^{*} N_{j 3}-N_{i 4}^{*} N_{j 4}\right), \quad H_{i j}^{R}=-\left(H_{i j}^{L}\right)^{*}=-H_{j i}^{L} \\
D_{i j}^{L}=s_{\beta} U_{i 1}^{*} V_{j 2}^{*}+c_{\beta} U_{i 2}^{*} V_{j 1}^{*}, \quad D_{i j}^{R}=s_{\beta} V_{i 2} U_{j 1}+c_{\beta} V_{i 1} U_{j 2}=\left(D^{L \dagger}\right)_{i j} \\
D_{i j}^{\prime L}=\left(N_{j 2}^{*}-t_{W} N_{j 1}^{*}\right)\left(N_{i 3}^{*} c_{\beta}-N_{i 4}^{*} s_{\beta}\right)+(i \leftrightarrow j), \quad D_{i j}^{\prime R}=\left(D_{i j}^{\prime L}\right)^{*} .
\end{gathered}
$$

Here, index 3(4) implies $H_{d}\left(H_{u}\right)$ following Ref. [2]. In the MSSM away from the split supersymmetry limit, Higgs boson couplings are modified as the relation $\tan \alpha=\tan \beta$ does not generally hold. For the lightest Higgs boson $H_{1}$, the couplings can be obtained by substituting $h \rightarrow H_{1}$ and $s_{\beta}\left(c_{\beta}\right) \rightarrow-c_{\alpha}\left(s_{\alpha}\right)$, where $s_{\beta}$ and $c_{\beta}$ are explicitly listed in the above equations for $D_{i j}^{L, R}$.

$C P$ and P-odd form factors are conventionally written as below [9] for incoming $V_{\mu}(q)$ (or $\left.h(q)\right)$ and outgoing $W_{\alpha}^{-}\left(p_{1}\right)$ and $W_{\beta}^{+}\left(p_{2}\right)$ (or $V_{\mu}\left(p_{1}\right)$ and $\left.V_{\nu}\left(p_{2}\right)\right)$

$$
\begin{aligned}
\Gamma_{\mathrm{WWV}}^{\mu \alpha \beta}= & i g_{W W V}\left[f_{6}^{V}(q) \epsilon^{\mu \alpha \beta \nu} q_{\nu}+\frac{f_{7}^{V}(q)}{M_{W}^{2}}\left(p_{1}-p_{2}\right)^{\mu} \epsilon^{\alpha \beta \rho \sigma} q_{\rho}\left(p_{1}-p_{2}\right)_{\sigma}+i f_{4}^{V}(q)\left(q^{\alpha} g^{\mu \beta}+q^{\beta} g^{\mu \alpha}\right)\right] \\
\Gamma_{H_{i} V V}^{\mu \nu}= & g M_{W}\left[g_{H_{i} W W}\left(S_{i}^{W}(q)\left(g_{\mu \nu}-\frac{2 p_{1 \mu} p_{2 \nu}}{M_{W}^{2}}\right)+\frac{P_{i}^{W}(q)}{M_{W}^{2}} \epsilon_{\mu \nu \alpha \beta} p_{1}^{\alpha} p_{2}^{\beta}\right)+\frac{1}{2 c_{W}^{2}} g_{H_{i} Z Z}\left(S_{i}^{Z}(q)\left(g_{\mu \nu}-\frac{2 p_{1 \mu} p_{2 \nu}}{M_{W}^{2}}\right)\right.\right. \\
& \left.\left.+\frac{P_{i}^{Z}(q)}{M_{W}^{2}} \epsilon_{\mu \nu \alpha \beta} p_{1}^{\alpha} p_{2}^{\beta}\right)\right],
\end{aligned}
$$

where $f_{6}^{V}=\tilde{\kappa}_{V}-\tilde{\lambda}_{V}, f_{7}^{V}=-\frac{1}{2} \tilde{\lambda}_{V}, f_{4}^{V}=g_{4}^{V}, P_{i}^{V}=\tilde{\eta}_{i}^{V}$. $g_{W W \gamma}=-e, u g_{W W Z}=-e \cot \theta_{W}$ and $g_{H_{i} V V}$ is the ratio of the $C P$-even $H_{i} V V$ coupling to the $\mathrm{SM} H_{i} V V$ coupling. $C P$-even form factor $S_{i}^{V}$ and C-odd form factor $f_{4}^{V}$ are shown for reference. More information about these form factors and the effective Lagrangian Eq. (1) can be found in [9].

\section{Triple boson vertices}

We represent triple gauge boson form factors first. These are generated via chargino/neutralino as shown in Fig. 3. The effective couplings are obtained in the limit of the onshell center-of-mass energy $s=q^{2} \rightarrow M_{V, H_{i}}^{2}$. For reference we list all three types of $C P$-violating $W W V$ couplings in terms of loop functions $a_{i}^{W W V}$.

$$
\begin{aligned}
f_{6}^{Z++}= & \frac{g^{2}}{16 \pi^{2} c_{W}^{2}} \sum_{i, j, k}\left[m_{i}^{+} m_{k}^{+} \operatorname{Im}\left(C_{j i}^{R *} C_{j k}^{R} F_{k i}^{L}-L\right) a_{1}^{W W Z}\right. \\
& +2 m_{i}^{+} m_{j}^{0} \operatorname{Im}\left(C_{j i}^{L *} C_{j k}^{R} F_{k i}^{R}-L\right) a_{2}^{W W Z} \\
& +2 \operatorname{Im}\left(C_{j i}^{R *} C_{j k}^{R} F_{k i}^{R}-L\right)\left\{M_{W}^{2} a_{5}^{W W Z}\right. \\
& \left.\left.+q^{2}\left(a_{4}^{W W Z} / 2-a_{6}^{W W Z}\right)+3 a_{8}^{W W Z}\right\}\right],
\end{aligned}
$$

$$
\begin{aligned}
f_{4}^{Z++}= & \frac{g^{2}}{16 \pi^{2} c_{W}^{2}} \sum_{i, j, k}\left[m_{i}^{+} m_{k}^{+} \operatorname{Im}\left(C_{j i}^{R *} C_{j k}^{R} F_{k i}^{L}+L\right) a_{1}^{W W Z}\right. \\
& +2 m_{i}^{+} m_{j}^{0} \operatorname{Im}\left(C_{j i}^{L *} C_{j k}^{R} F_{k i}^{R}+L\right) a_{3}^{W W Z} \\
& +2 \operatorname{Im}\left(C_{j i}^{R *} C_{j k}^{R} F_{k i}^{R}+L\right)\left\{M_{W}^{2} a_{5}^{W W Z}\right. \\
& \left.\left.-q^{2} a_{6}^{W W Z}+3 a_{8}^{W W Z}\right\}\right],
\end{aligned}
$$




$$
\begin{array}{rlr}
f_{6}^{Z 00}= & \frac{g^{2}}{16 \pi^{2} c_{W}^{2}} \sum_{i, j, k}\left[m_{i}^{0} m_{k}^{0} \operatorname{Im}\left(C_{i j}^{R *} C_{k j}^{R} H_{i k}^{L}-L\right) a_{1}^{W W Z}\right. & f_{6}^{\gamma}=\frac{e^{2}}{8 \pi^{2}} \sum_{i, j} m_{i}^{+} m_{j}^{0} \operatorname{Im}\left(C_{j i}^{L *} C_{j i}^{R}-L\right) a_{2}^{W W \gamma}, \\
& +2 m_{j}^{+} m_{k}^{0} \operatorname{Im}\left(C_{i j}^{L *} C_{j k}^{R} H_{i k}^{L}-L\right) a_{2}^{W W Z} \\
& +2 \operatorname{Im}\left(C_{i j}^{R *} C_{k j}^{R} H_{i k}^{R}-L\right)\left\{M_{W}^{2} a_{5}^{W W Z}\right. \\
& \left.\left.+q^{2}\left(a_{4}^{W W Z} / 2-a_{6}^{W W Z}\right)+3 a_{8}^{W W Z}\right\}\right], \quad \text { (A8) } \quad f_{4}^{\gamma}=f_{7}^{\gamma}=0 .
\end{array}
$$$$
f_{4}^{Z 00}=\frac{g^{2}}{16 \pi^{2} c_{W}^{2}} \sum_{i, j, k}\left[-m_{i}^{0} m_{k}^{0} \operatorname{Im}\left(C_{i j}^{R *} C_{k j}^{R} H_{i k}^{L}+L\right) a_{1}^{W W Z}\right.
$$$$
+2 m_{j}^{+} m_{k}^{0} \operatorname{Im}\left(C_{i j}^{L *} C_{k j}^{R} H_{i k}^{L}+L\right) a_{3}^{W W Z}
$$$$
+2 \operatorname{Im}\left(C_{i j}^{R *} C_{k j}^{R} H_{k i}^{R}+L\right)\left\{M_{W}^{2} a_{5}^{W W Z}\right.
$$$$
\left.\left.+q^{2} a_{6}^{W W Z}-3 a_{8}^{W W Z}\right\}\right] \text {, }
$$

$$
f_{7}^{Z}=0
$$

Subscript ${ }^{++}\left({ }^{00}\right)$ implies the contributions from the first (second) diagram in Fig. 3 where two charginos (neutralinos) are running in the loop. $L$ inside the Im part implies the same coupling combination with $L \leftrightarrow R$. $f_{4}^{\gamma}$ is zero because $W W \gamma$ form factors define the electric charge of the $W$ boson in the Coulomb limit, while C-odd parts flip the electric charge.

The loop functions are given as (assuming light on-shell bosons)

$$
\begin{aligned}
& a_{i}^{W W Z}=\int_{0}^{1} d x \int_{0}^{1-x} d y \frac{b_{i}}{\left(m_{i}^{2}-m_{j}^{2}\right) x+\left(m_{k}^{2}-m_{j}^{2}\right) y+m_{j}^{2}-q^{2} x y} \quad \text { for } i=1, \cdots, 7 \\
& a_{8}^{W W Z}=\int_{0}^{1} d x \int_{0}^{1-x} d y(y-x) \cdot \ln \left(\left(m_{i}^{2}-m_{j}^{2}\right) x+\left(m_{k}^{2}-m_{j}^{2}\right) y+m_{j}^{2}-q^{2} x y\right)=\left(m_{k}^{2}-m_{i}^{2}\right) a_{7}^{W W Z,}
\end{aligned}
$$

where $q$ is incoming $Z$ boson momentum. $a_{2}^{W W \gamma}$ can be obtained by taking $m_{k}=m_{i}$ in $a_{2}^{W W Z}$. Coefficients $b_{i}$ are given as

$$
\begin{gathered}
b_{1}=x-y, \quad b_{2}=y-x+1, \quad b_{3}=x+y-1, \quad b_{4}=(y-x)(x+y-1) \\
b_{5}=(y-x)(x+y-1)^{2}, \quad b_{6}=(y-x) x y, \quad b_{7}=x y .
\end{gathered}
$$

These results numerically match well with previous computations [37].

In a similar way, $H_{i} V V$ couplings are generated via chargino/neutralino and top/bottom quarks (not in split supersymmetry). Here, we represent only $C P$-odd $h Z Z$ and $h W W$ couplings as these are relevant for our numerical studies. These are given in terms of loop functions $c_{i}$

$$
\begin{aligned}
P_{h}^{Z} \cdot g_{h V V}= & \frac{\sqrt{2} \alpha M_{W}}{\pi s_{W}^{2}} \sum_{i, j, k=1}^{2}\left(m_{i} \operatorname{Im}\left(F_{j i}^{R} D_{i k}^{R} F_{k j}^{R}-L\right) c_{1}(i, j, k)+m_{j} \operatorname{Im}\left(F_{j i}^{R} D_{i k}^{L} F_{k j}^{L}-L\right) c_{2}(i, j, k)\right) \\
& +\frac{\sqrt{2} \alpha M_{W}}{\pi s_{W}^{2}} \sum_{i, j, k=1}^{4}\left(m_{i} \operatorname{Im}\left(H_{j i}^{R} D_{i k}^{\prime R} H_{k j}^{R}-L\right) c_{1}(i, j, k)+m_{j} \operatorname{Im}\left(H_{j i}^{R} D_{i k}^{\prime L} H_{k j}^{L}-L\right) c_{2}(i, j, k)\right) \\
& +\frac{3 \alpha M_{W}}{\pi s_{W}^{2}} \sum_{f=t, b} m_{f}\left(\operatorname{Im}\left(F_{Z}^{R} D_{f}^{R} F_{Z}^{R}-L\right) c_{3}(f)+\operatorname{Im}\left(F_{Z}^{R} D_{f}^{L} F_{Z}^{L}-L\right) c_{4}(f)\right), \\
P_{h}^{W} \cdot g_{h V V}= & \frac{\sqrt{2} \alpha M_{W}}{\pi s_{W}^{2}} \sum_{i, j, k=1}^{2}\left(m_{i} \operatorname{Im}\left(C_{j i}^{R} D_{i k}^{R} C_{k j}^{* R}-L\right) c_{1}(i, j, k)+m_{j} \operatorname{Im}\left(C_{j i}^{R} D_{i k}^{L} C_{k j}^{* L}-L\right) c_{2}(i, j, k)\right)+\frac{\sqrt{2} \alpha M_{W}}{\pi s_{W}^{2}} \\
& \times \sum_{i, j, k=1}^{4}\left(m_{i} \operatorname{Im}\left(C_{j i}^{* R} D_{i k}^{\prime R} C_{k j}^{R}-L\right) c_{1}(i, j, k)+m_{j} \operatorname{Im}\left(C_{j i}^{* R} D_{i k}^{\prime L} C_{k j}^{L}-L\right) c_{2}(i, j, k)\right) \\
& +\frac{3 \alpha M_{W}}{\pi s_{W}^{2}} \sum_{f=t, b} m_{f} \Gamma \operatorname{Im}\left(-D_{f}^{L}\right) c_{3}(f),
\end{aligned}
$$

where couplings with quarks are given as 


$$
\begin{gathered}
F_{Z}^{L, R}=T_{f}^{3}-Q_{f} s_{W}^{2}, \quad D_{t}^{L}=\frac{m_{t}}{M_{W} s_{\beta}}\left(O_{H_{u} i}+i O_{A i} c_{\beta}\right), \quad D_{t}^{R}=\left(D_{t}^{L}\right)^{*} \\
D_{b}^{L}=\frac{m_{b}}{M_{W} c_{\beta}}\left(O_{H_{d} i}+i O_{A i} s_{\beta}\right), \quad D_{b}^{R}=\left(D_{b}^{L}\right)^{*} .
\end{gathered}
$$

In order to see the dependence on $C P$ even-odd mixing better, we simplify the quark contributions in the third lines by approximately treating $s_{W}^{2} \approx 0.25$. These quark contributions are given as

$$
\begin{aligned}
& P_{h}^{Z} \cdot g_{h V V} \cong-\frac{3 \alpha O_{A 1}}{\pi s_{W}^{2}}\left\{\frac{m_{t}^{2}}{t_{\beta}}\left(\frac{10}{72} c_{3}(t)+\frac{8}{72} c_{4}(t)\right)+m_{b}^{2} t_{\beta}\left(\frac{13}{72} c_{3}(b)+\frac{5}{72} c_{4}(b)\right)\right\}+\cdots \\
& P_{h}^{W} \cdot g_{h V V} \cong-\frac{3 \alpha O_{A 1}}{\pi s_{W}^{2}}\left\{\frac{m_{t}^{2}}{t_{\beta}} c_{3}(t)+m_{b}^{2} t_{\beta} c_{3}(b)\right\}+\cdots
\end{aligned}
$$

We can see that the $C P$-odd $h Z Z$ coupling is very sensitive to the $C P$ even-odd mixing $O_{A 1}$ and $t_{\beta}$. Quantum corrections to the $C P$-even couplings are ignored as they are much smaller than the tree-level values.

The loop functions are (assuming on-shell vector bosons)

$$
\begin{aligned}
c_{1}(i, j, k) & =\int_{0}^{1} d x \int_{0}^{1-x} d y \frac{x+y}{\left(m_{i}^{2}-m_{j}^{2}\right) x+\left(m_{k}^{2}-m_{j}^{2}\right) y+m_{j}^{2}+M_{V}^{2}(x+y)(x+y-1)-q^{2} x y} \\
c_{2}(i, j, k) & =\int_{0}^{1} d x \int_{0}^{1-x} d y \frac{x+y-1}{\left(m_{i}^{2}-m_{j}^{2}\right) x+\left(m_{k}^{2}-m_{j}^{2}\right) y+m_{j}^{2}+M_{V}^{2}(x+y)(x+y-1)-q^{2} x y} \\
c_{3}(f) & =\int_{0}^{1} d x \int_{0}^{1-x} d y \frac{x+y}{m_{f}^{2}+M_{V}^{2}(x+y)(x+y-1)-q^{2} x y} \\
c_{4}(f) & =\int_{0}^{1} d x \int_{0}^{1-x} d y \frac{x+y-1}{m_{f}^{2}+M_{V}^{2}(x+y)(x+y-1)-q^{2} x y},
\end{aligned}
$$

where $q$ is Higgs momentum.

In this paper, we use on-shell (constant) couplings rather than considering full momentum dependence. This momentum dependence comes from integrating out dynamical degrees of freedom, and are shown in Fig. 8. Couplings around the threshold region are different from on-shell couplings. However, the typical energy scales of LHC

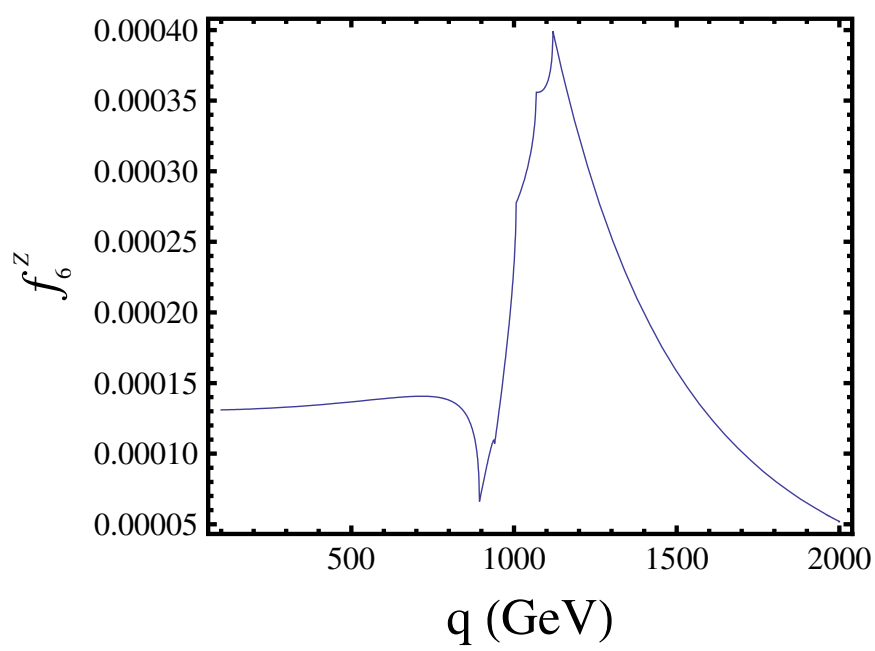

processes that care applicability in the measurement of TBVs are only about $200 \mathrm{GeV}$ as shown in Fig. 9. The on-shell coupling thus may contribute more to the crosssection support than the threshold behavior. Figure 8 also shows that the maximum couplings in the threshold region are only $\mathcal{O}(1)$ factor larger than the on-shell couplings. Although the threshold behavior depends on input parame-

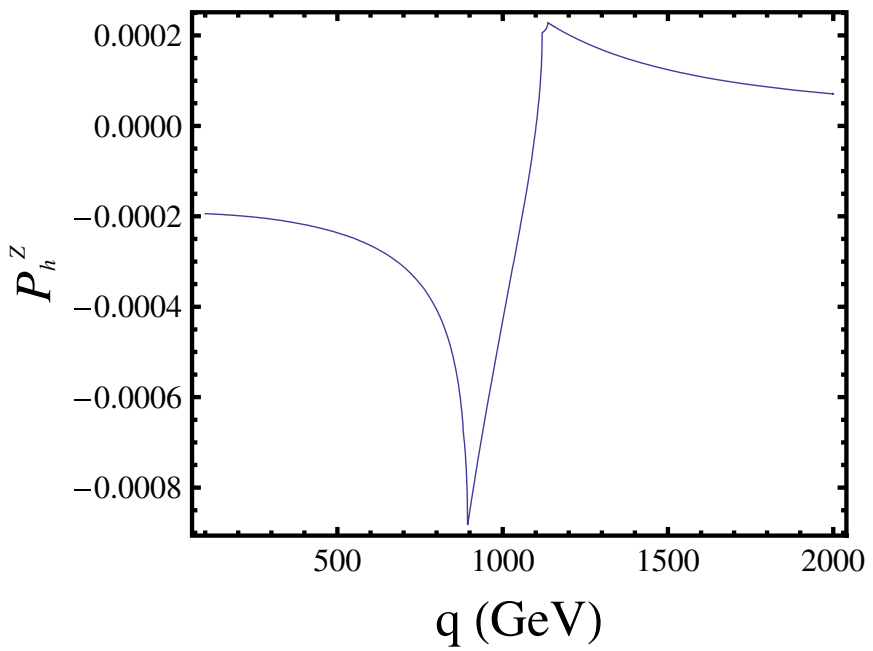

FIG. 8 (color online). Sample plots show the momentum dependence of form factors $f_{6}^{Z}$ (left) and $P_{h}^{Z}$ (right) in split supersymmetry. $q$ is $Z$ or Higgs momentum. $M_{1}=M_{2}=\mu=500 \mathrm{GeV}$ and $t_{\beta}=1$ are used. 

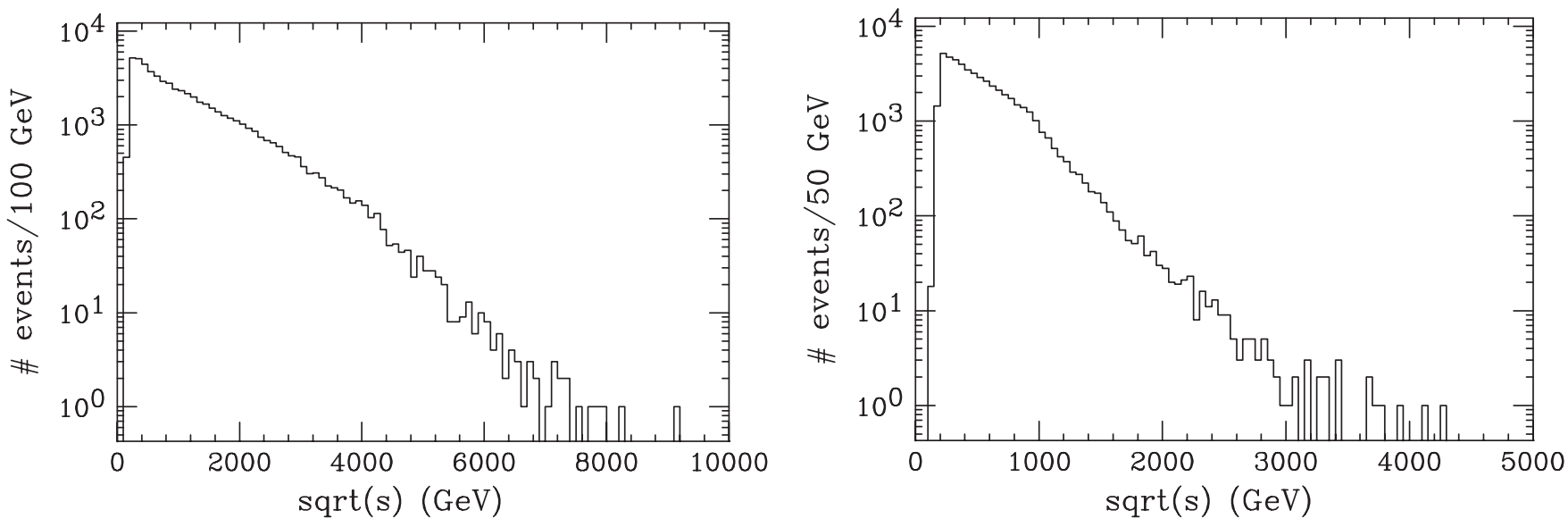

FIG. 9. Sample center-of-mass energy $\sqrt{s}$ distributions of $p p \rightarrow W^{*} \rightarrow W Z$ (left) and $p p \rightarrow h \rightarrow Z Z$ (right) in which collider sensitivities of TBVs are usually studied in previous literature.

ters, we checked that maximum couplings are larger than the on-shell couplings by at most $\mathcal{O}(10)$ factor, which does not affect our conclusion. It is also convenient to use onshell couplings since it facilitates the comparison of our result with previous collider studies of TBVs that usually assume constant couplings.

\section{APPENDIX B: ELECTRIC DIPOLE MOMENTS}

EDM is a parity and time-reversal violating electromagnetic property of a fermion at the fermion mass scale. In field theory language, EDM comes from the $C P$-odd lowenergy effective operator $-i 1 / 2 \bar{f} \sigma_{\mu \nu} \gamma_{5} f F^{\mu \nu}$ with onshell fermion $f$ and a photon. Exact full two-loop calculations have been carried out in [22-24] for split supersymmetry, and in [32-34] for the MSSM with one-loop EDM suppressed. In this appendix, we rather compute eEDM in split supersymmetry by inserting effective $C P$-odd TBVs into relevant diagrams in Fig. 2. We work in dimensional regularization and $\overline{M S}$ scheme. It is a good way to check the previously computed results. For more accurate numerical analysis, we use the full two-loop results.

For reference, we list the leading order EDM in split supersymmetry (in the limit $M_{1}, M_{2}, \mu \gg M_{W}, M_{h}$ ) calculated using effective couplings

$$
\begin{aligned}
d_{f}^{W W}= & -\frac{e \alpha^{2} T_{f}}{8 \pi^{2} s_{W}^{4}} \sum_{i, k} \frac{m_{f} m_{i}^{+} m_{k}^{0}}{M_{W}^{2}} \operatorname{Im}\left(C_{k i}^{* L} C_{k i}^{R}\right) \cdot \frac{1}{m_{i}^{2}-m_{k}^{2}} \\
& \times\left(\frac{m_{k}^{2}}{m_{i}^{2}-m_{k}^{2}} \ln \frac{m_{k}^{2}}{m_{i}^{2}}+1\right) \cdot\left(\ln \frac{\mu^{2}}{M_{W}^{2}}+\frac{3}{2}\right),
\end{aligned}
$$

$$
d_{f}^{\gamma h}=\frac{e Q_{f} \alpha^{2}}{4 \sqrt{2} \pi^{2} s_{W}^{2}} \sum_{i} \operatorname{Im}\left(D_{i i}^{R}\right) \frac{m_{f}}{M_{W} m_{i}^{+}}\left(\frac{1}{2} \ln \frac{\mu^{2}}{M_{h}^{2}}+\frac{3}{4}\right)
$$

$$
\begin{aligned}
d_{f}^{Z h}= & -\frac{e \alpha^{2}\left(T_{f}^{3}-2 Q_{f} s_{W}^{2}\right)}{8 \sqrt{2} c_{W}^{2} \pi^{2} s_{W}^{4}} \sum_{i, j} \frac{m_{f} m_{i}^{+}}{M_{W}} \operatorname{Im}\left(D_{i j}^{R} F_{j i}^{R}\right. \\
& \left.-D_{i j}^{L} F_{j i}^{L}\right) \cdot \frac{1}{m_{i}^{2}-m_{j}^{2}}\left(1-\frac{m_{j}^{2}}{m_{i}^{2}-m_{j}^{2}} \ln \frac{m_{i}^{2}}{m_{j}^{2}}\right) \\
& \cdot \frac{1}{2}\left(\log \frac{\mu^{2}}{M_{h}^{2}}+\frac{M_{Z}^{2}}{M_{h}^{2}-M_{Z}^{2}} \ln \frac{M_{Z}^{2}}{M_{h}^{2}}\right),
\end{aligned}
$$

where superscripts imply two particles that mediate $C P$ violation to SM fermions. When two inos running in the loop are (almost) degenerate, these formula simplify as the following:

$$
\begin{aligned}
d_{f}^{W W}= & -\frac{e \alpha^{2} T_{f}}{8 \pi^{2} s_{W}^{4}} \sum_{i, k} \frac{m_{f} m_{k}^{0}}{M_{W}^{2} m_{i}^{+}} \operatorname{Im}\left(C_{k i}^{* L} C_{k i}^{R}\right) \cdot\left(\ln \frac{\mu^{2}}{M_{W}^{2}}+\frac{3}{2}\right), \\
d_{f}^{Z h}= & -\frac{e \alpha^{2}\left(T_{f}^{3}-2 Q_{f} s_{W}^{2}\right)}{8 \sqrt{2} c_{W}^{2} \pi^{2} s_{W}^{4}} \sum_{i, j} \frac{m_{f}}{M_{W} m_{i}^{+}} \operatorname{Im}\left(D_{i j}^{R} F_{j i}^{R}\right. \\
& \left.-D_{i j}^{L} F_{j i}^{L}\right) \cdot \frac{1}{2}\left(\ln \frac{\mu^{2}}{M_{h}^{2}}+\frac{M_{Z}^{2}}{M_{h}^{2}-M_{Z}^{2}} \ln \frac{M_{Z}^{2}}{M_{h}^{2}}\right) .
\end{aligned}
$$

We checked that our results agree with the most recent calculations of [33].

Effective matching scale $\mu$ may be chosen to obtain the EDM numerically close to the full two-loop result $[18,22]$

$$
\begin{gathered}
\mu^{2}=m_{\chi_{1}^{+}} m_{\chi_{2}^{+}}, \quad m_{\chi_{1}^{+}} m_{\chi_{2}^{+}}, \\
m_{\chi_{1}^{+}} m_{\chi_{4}^{0}} \text { for } \gamma h, Z h, W W, \text { respectively. }
\end{gathered}
$$

We used the following relations, which follow from unitarity and the definitions of mixing matrices, to reach the final form

$$
\begin{aligned}
\operatorname{Im}\left(D_{i j}^{R} F_{j i}^{R}\right) m_{i}^{+} & =\operatorname{Im}\left(D_{j i}^{R} F_{i j}^{R}\right) m_{j}^{+} \text {no sum } \\
\operatorname{Im}\left(D_{i j}^{R} F_{j i}^{L}\right) m_{j}^{+} & =-\operatorname{Im}\left(D_{i j}^{L} F_{j i}^{L}\right) m_{i}^{+} \quad \text { and } \quad R \leftrightarrow L .
\end{aligned}
$$


[1] B. C. Regan et al., Phys. Rev. Lett. 88, 071805 (2002).

[2] S.P. Martin, arXiv:hep-ph/9709356.

[3] S. Abel, S. Khalil, and O. Lebedev, Nucl. Phys. B606, 151 (2001).

[4] D. A. Demir, O. Lebedev, K. A. Olive, M. Pospelov, and A. Ritz, Nucl. Phys. B680, 339 (2004).

[5] M. Brhlik, G. J. Good, and G. L. Kane, Phys. Rev. D 59, 115004 (1999).

[6] T. Ibrahim and P. Nath, Phys. Rev. D 57, 478 (1998); 58, 019901(E) (1998); 60, 079903(E) (1999);

[7] T. Ibrahim and P. Nath, Phys. Rev. D 58, 111301 (1998); 60, 099902(E) (1999).

[8] J. Kumar, A. Rajaraman, and J. D. Wells, Phys. Rev. D 78, 035014 (2008).

[9] K. Hagiwara, R. D. Peccei, D. Zeppenfeld, and K. Hikasa, Nucl. Phys. B282, 253 (1987).

[10] B. Ananthanarayan, S. D. Rindani, R. K. Singh, and A. Bartl, Phys. Lett. B 593, 95 (2004); 608, 274(E) (2005); M. Diehl, O. Nachtmann, and F. Nagel, Eur. Phys. J. C 32, 17 (2003); D. Choudhury, J. Kalinowski, and A. Kulesza, Phys. Lett. B 457, 193 (1999); A. A. Likhoded, G. Valencia, and O.P. Yushchenko, Phys. Rev. D 57, 2974 (1998); D. Choudhury and S. D. Rindani, Phys. Lett. B 335, 198 (1994); B. Ananthanarayan and S. D. Rindani, Phys. Rev. D 70, 036005 (2004); V. D. Barger, T. Falk, T. Han, J. Jiang, T. Li, and T. Plehn, Phys. Rev. D 64, 056007 (2001); P. Langacker, G. Paz, L. T. Wang, and I. Yavin, J. High Energy Phys. 07 (2007) 055.

[11] R. M. Godbole, D. J. Miller, and M. M. Muhlleitner, J. High Energy Phys. 12 (2007) 031; C. P. Buszello, P. Marquard, and J.J. van der Bij, arXiv:hep-ph/0406181; E. Accomando et al., arXiv:hep-ph/0608079; T. Han and J. Jiang, Phys. Rev. D 63, 096007 (2001); S. S. Biswal, R. M. Godbole, R. K. Singh, and D. Choudhury, Phys. Rev. D 73, 035001 (2006); 74, 039904(E) (2006); R. M. Godbole, S. Kraml, S. D. Rindani, and R. K. Singh, Phys. Rev. D 74, 095006 (2006); 74, 119901(E) (2006).

[12] S. Weinberg, Phys. Rev. Lett. 63, 2333 (1989).

[13] D. Kawall, F. Bay, S. Bickman, Y. Jiang, and D. DeMille, Phys. Rev. Lett. 92, 133007 (2004).

[14] J. J. Hudson, B. E. Sauer, M. R. Tarbutt, and E. A. Hinds, Phys. Rev. Lett. 89, 023003 (2002).

[15] C. Y. Liu and S. K. Lamoreaux, Mod. Phys. Lett. A 19, 1235 (2004).

[16] N. Arkani-Hamed and S. Dimopoulos, J. High Energy Phys. 06 (2005) 073.
[17] G.F. Giudice and A. Romanino, Nucl. Phys. B699, 65 (2004); B706, 65(E) (2005).

[18] N. Arkani-Hamed, S. Dimopoulos, G. F. Giudice, and A. Romanino, Nucl. Phys. B709, 3 (2005).

[19] J.D. Wells, arXiv:hep-ph/0306127; Phys. Rev. D 71, 015013 (2005).

[20] S. Dimopoulos and S. D. Thomas, Nucl. Phys. B465, 23 (1996).

[21] M. Dugan, B. Grinstein, and L. J. Hall, Nucl. Phys. B255, 413 (1985).

[22] G. F. Giudice and A. Romanino, Phys. Lett. B 634, 307 (2006).

[23] D. Chang, W. F. Chang, and W. Y. Keung, Phys. Rev. D 71, 076006 (2005).

[24] T.F. Feng, L. Sun, and X. Y. Yang, Phys. Rev. D 77, 116008 (2008).

[25] P. G. Harris et al., Phys. Rev. Lett. 82, 904 (1999).

[26] S. Abel and O. Lebedev, J. High Energy Phys. 01 (2006) 133.

[27] S. Dimopoulos and G. F. Giudice, Phys. Lett. B 357, 573 (1995); A. G. Cohen, D. B. Kaplan, and A.E. Nelson, Phys. Lett. B 388, 588 (1996).

[28] A. Pilaftsis, Phys. Rev. D 58, 096010 (1998).

[29] A. Pilaftsis, Phys. Lett. B 435, 88 (1998).

[30] A. Pilaftsis, in International Symposium on Particles, Strings and Cosmology (PASCOS 1999). Lake Tahoe California, December 10-16, 1999, edited by K. Cheung, J.F Gunion, and S. Mrenna (World Scientific, Singapore, 2000) p. 164.

[31] D. A. Demir, Phys. Rev. D 60, 055006 (1999).

[32] A. Pilaftsis, Nucl. Phys. B644, 263 (2002).

[33] Y. Li, S. Profumo, and M. Ramsey-Musolf, Phys. Rev. D 78, 075009 (2008).

[34] T. H. West, Phys. Rev. D 50, 7025 (1994); T. Kadoyoshi and N. Oshimo, Phys. Rev. D 55, 1481 (1997).

[35] J. S. Lee, M. Carena, J. Ellis, A. Pilaftsis, and C.E. M. Wagner, Comput. Phys. Commun. 180, 312 (2009); J. S. Lee, A. Pilaftsis, M. S. Carena, S. Y. Choi, M. Drees, J. R. Ellis, and C. E. M. Wagner, Comput. Phys. Commun. 156, 283 (2004); The program can be found at http://www.hep. man.ac.uk/u/jslee/CPsuperH.html.

[36] K. S. Babu, C. F. Kolda, J. March-Russell, and F. Wilczek, Phys. Rev. D 59, 016004 (1998).

[37] M. Kitahara, M. Marui, N. Oshimo, T. Saito, and A. Sugamoto, Eur. Phys. J. C 4, 661 (1998). 